\title{
Detecção de casos novos de hanseníase no município de Prudentópolis, PR: uma análise de 1998 a 2005
}

\author{
Detection of new cases of leprosy in the City of Prudentópolis, PR: \\ the analysis from 1998 to 2005
}

\author{
Lucas Augusto Thomé Sanches ${ }^{1}$, Elaine Pittner ${ }^{1}$, Hermes Francisco Sanches ${ }^{1}$ \\ e Marta Chagas Monteiro ${ }^{1}$
}

\begin{abstract}
RESUMO
Este trabalho teve como objetivo detectar os casos novos de hanseníase do período de 1998 a 2005 no município de Prudentópolis, PR. Assim como, verificar a faixa etária e a forma clínica predominante entre os casos de hanseníase. Os dados foram obtidos através das fichas clínicoepidemiológicas de casos com diagnóstico definitivo de hanseníase notificado no Sistema de Informação sobre Agravos de Notificação, de 1998 a 2005. Neste periodo, foram relatados 222 casos de hanseníase, dos quais 63\% apresentavam a forma multibacilar, com predominância da forma clínica virchowiana, indicando um processo de intensa transmissão da doença. Dos casos de hanseníase, 35\% dos pacientes se encontravam na faixa economicamente ativa, 31 a 45 anos. Esses indicadores apontam para uma elevada circulação do bacilo na comunidade, já que um fator de risco para infecção pelo bacilo Mycobacterium Leprae é o contato com pacientes com a forma multibacilar, sem tratamento, embora nas duas últimas décadas, tenha havido redução drástica da prevalência da hanseníase pela implementação da poliquimioterapia e outras medidas preventivas.
\end{abstract}

Palavra-chaves: Hanseníase. Forma multibacilar. Detecção de casos novos.

\begin{abstract}
The aim of this study was to evaluate the detection of new cases of leprosy in Prudentópolis, State of Paraná, from 1998 to 2005. Additionally, we investigated the age group and predominant clinical form of leprosy cases. Data were obtained from clinical-epidemiological records of confirmed leprosy cases that were notified in the Information System for Notifiable Diseases, between 1998 and 2005. Over this period, 222 leprosy cases were notified, of which 63\% presented the multibacillary form, with predominance of the Virchow clinical form, thus indicating a process of intense disease transmission. Among these leprosy cases, 35\% of the patients were at an economically active age (31 to 45 years old). These indicators showed that there was a high level of bacillus circulation in the community, considering that one risk factor for infection by the bacillus Mycobacterium leprae is contact with untreated individuals who have the multibacillary form. This is despite the drastic reduction in leprosy prevalence over the last two decades, through the implementation of polychemotherapy and other preventive measures.
\end{abstract}

Key-words: Leprosy. Multibacillary form. Detection of new cases.

A hanseníase, ou mal de hansen (MH), causada pelo Mycobacterium leprae ${ }^{3}$, constitui um relevante problema de saúde pública no Brasil, onde o controle desta endemia representa um verdadeiro desafio, a apresentar o segundo maior número de casos registrados ( 42.055 casos incidentes), atrás apenas da Índia com 537.956 casos $^{4}$. Considerada uma doença polimorfa, a expressão das manifestações clínicas da hanseníase reflete a relação entre o hospedeiro e o parasita. Os indivíduos adoecem de acordo com a resposta imunológica específica frente ao bacilo. Para Bechelli e cols ${ }^{4}$, existe uma forte correlação entre idade e forma clínica, destacando que a ocorrência de forma multibacilares (virchowiana e dimorfa) aumenta com a idade.

Em 1985, a hanseníase foi considerada endêmica em 122 países a ao final do ano de 2000, o Brasil e outros 14 países ainda permaneciam nesta condição. A endemia hansênica apresenta-se, na virada do milênio, no limiar da sua eliminação como problema de saúde pública em nível mundial. 0 marco histórico dessa estratégia foi a resolução da $44^{\mathrm{a}}$ Assembléia

1. Departamento de Farmácia, Universidade Estadual do Centro-Oeste, Guarapuava, PR.

Endereço para correspondência: Prof ${ }^{a}$ Marta Chagas Monteiro. Depto de Farmácia/Centro Politécnico/UNICENTRO. R. Simeão Camargo Varela de Sá, 03. Cascavel, 85040-080, Guarapuava-PR

Tel: 5542 3629-8137; Fax: $55423629-8100$

e-mail: martachagas2@yahoo.com.br

Recebido para publicação em: 19/10/2006

Aceito em: 17/08/0207 
Mundial (1991), que definiu como eliminação da hanseníase a prevalência de registro abaixo de um caso para 10.000 habitantes, meta programática que deveria ter sido alcançada pelos países endêmicos até $2000^{20}$. As perspectivas de eliminação global da hanseníase dentro do contexto epidemiológico podem ser revistas em algumas publicações ${ }^{925}$.

No Brasil, o número de casos registrados foi aumentando até o ano de 1990, passando a declinar a partir de 1991, quando a poliquimioterapia (PQT) foi implantada em massa em todo o país ${ }^{8}$. A partir disto, as taxas de prevalência tem declinado no mundo ano a ano e a meta de eliminação vem sendo alcançada em vários países, resultado da consolidação do tratamento poliquimioterápico. Entretanto, no Brasil, as taxas de detecção de casos novos têm se mantido elevadas, e a expectativa era que se alcançasse a meta de eliminação da doença em 2005, quando a prevalência deveria ser inferior a 1,0/10.000 habitantes ${ }^{3}{ }^{11}$. No entanto, alguns estudos epidemiológicos vêm relatando as principais regiões no Brasil com altos índices de hanseníase, a fim de possibilitar que o Plano de Eliminação da Hanseníase possa ser mais eficiente nas áreas de maior prevalência5.

Neste sentido, o presente trabalho teve como objetivo detectar os casos novos de hanseníase, a faixa etária e a forma clínica predominante entre os pacientes com hanseníase no Município de Prudentópolis, Estado do Paraná, no período de 1998 a 2005.

\section{MATERIAL E MÉTODOS}

Foram analisadas 222 fichas clínico-epidemiológicas com diagnóstico definitivo de hanseníase notificado à Secretaria de Saúde do Estado e Secretaria Municipal de Prudentópolis, pelo Sistema de Informação sobre Agravos de Notificação (SINAM), de 1998 a 2005, considerando apenas os casos relativos aos residentes no Município de Prudentópolis, PR.

Nas fichas epidemiológicas, foram analisados os seguintes itens: a) idade do paciente, a qual foi separada nas seguintes faixas etária: crianças de zero a quinze anos (representam à evolução da doença no município); indivíduos de 16 a 30, 31-45 e 46-60 (população economicamente ativa) e indivíduos com mais de 61 anos (população não economicamente ativa); b) classificação operacional da doença: multibacilar ou paucibacilar e c) formas clínicas segundo a classificação de Madri (virchowiana, dimorfa, indeterminada e tuberculóide).

Os resultados foram avaliados através do teste de qui-quadrado considerando-se $\mathrm{p}<0,05$ como significância estatística, para avaliar as diferenças de proporção de detecção de casos novos por ano e também as proporções de números de pacientes em relação à classificação operacional e formas clínicas da doença.

\section{RESULTADOS}

De 1998 a 2005, foram registrados 222 casos de hanseníase na Secretaria Municipal de Saúde de Prudentópolis, com uma média de 27,7 casos novos de hanseníase por ano. Na Figura 1, observa-se que o ano de 2002 apresentou um aumento de pelo menos duas vezes no número de casos novos de hanseníase no município, 56 (25,2\%) casos, valores que foram significativos pelo teste de qui-quadrado quando comparados aos demais anos $\left(\chi^{2} \geq 4,60 ; \mathrm{p} \leq 0,05\right)$.

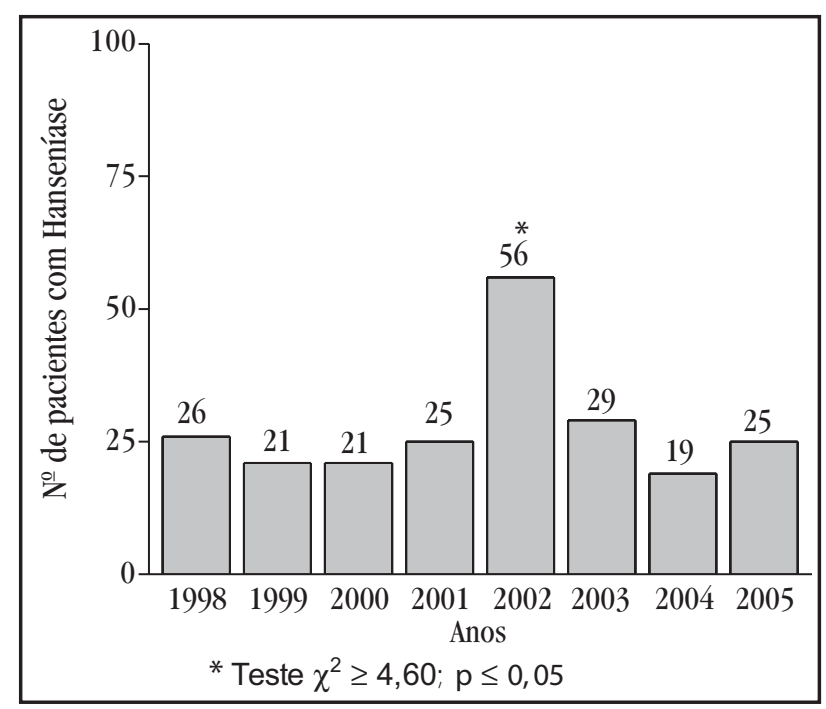

Figura 1 - Casos novos de hanseníase no município de PrudentópolisPR, 1998 a 2005.

Na Figura 2, observa-se que dos 222 casos novos de hanseníase, 139 (63\%) casos apresentavam a forma multibacilar e 83 (37\%) a forma paucibacilar, tendo a forma multibacilar como a forma da doença predominante no município $\left(\chi^{2}=7,47 ; \mathrm{p}<0.01\right)$. Dos casos pertencentes à forma multibacilar, 113 (51\%) casos foram diagnosticados como pertencentes à forma clínica virchowiana (Figura 3), seguida da dimorfa que apresentou 26 (12\%) casos. Quanto à forma paucibacilar, diagnosticou-se 56 casos com a forma clínica Indeterminada, correspondendo $25 \%$ do total e 27 (12\%) casos pertencentes à forma tuberculóide. Valores de qui-quadrado significativos $\left(\chi^{2} \geq 7,80, \mathrm{p} \leq 0.01\right)$ mostraram que a forma virchowiana foi à forma clínica predominante entre os pacientes com hanseníase (Figura 3).

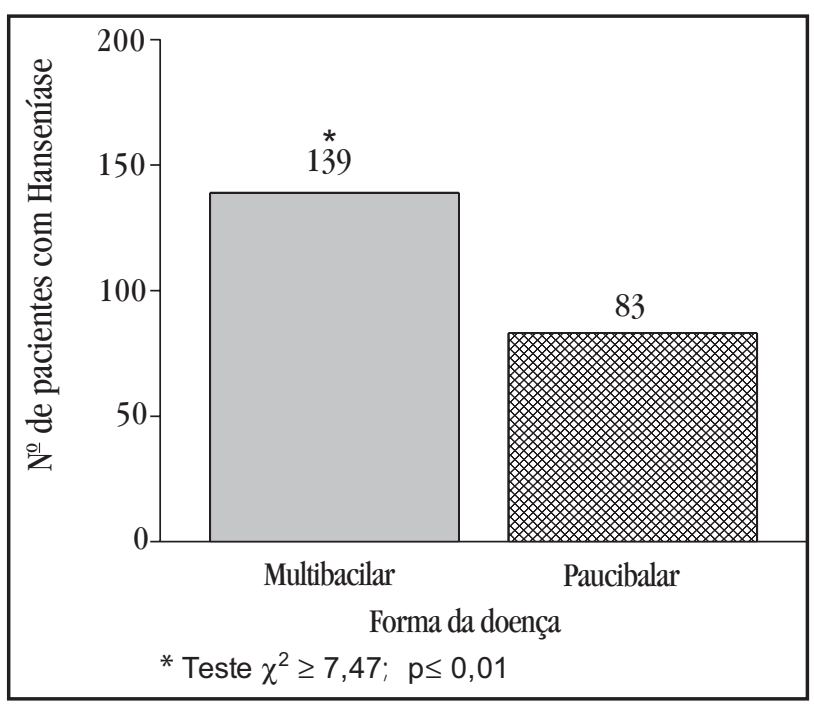

Figura 2 - Casos novos de hanseníase, segundo classificação operacional: Multibacilar e Paucipacilar, Prudentópolis-PR, 1998 a 2005. 


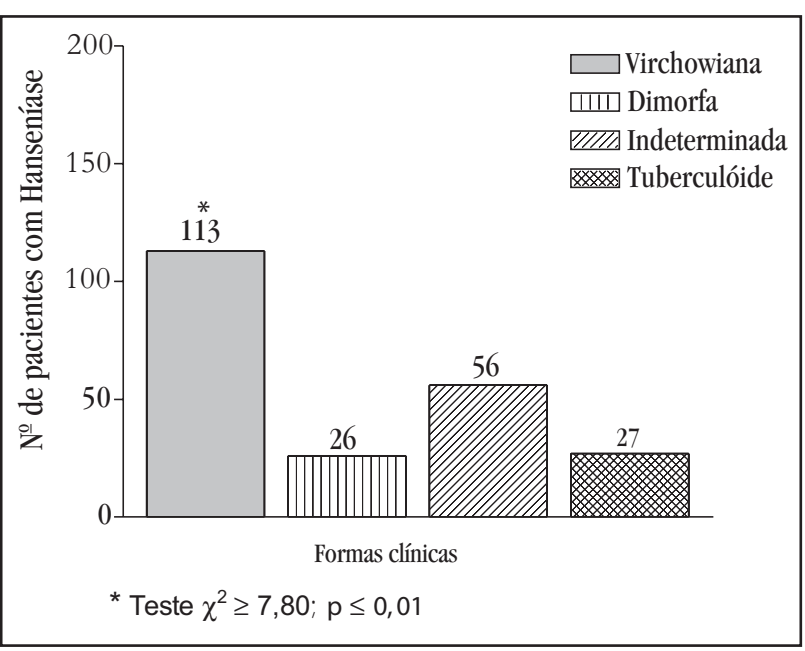

Figura 3 - Casos novos de hanseníase, segundo formas Clínicas (Classificação de Madri), Prudentópolis-PR, 1998 a 2005.

A Figura 4 mostra a predominância de casos novos de hanseníase por diferentes faixas etárias ( 0 a 15, 16-30, 31-45, 46-60 e mais de 61 anos), demonstrando que 35\% (77 casos) dos pacientes se encontravam entre 31 a 45 anos de idade, seguida das faixas etárias 46 a 60 (25\%) e 16 a 30 anos (21\%), indivíduos enquadrados na faixa economicamente ativa. Além disto, observa-se nessa mesma figura que somente 30 pacientes se encontravam com mais de 61 anos (13\%) e 12 (5\%) na faixa etária de 0 a 15 anos.

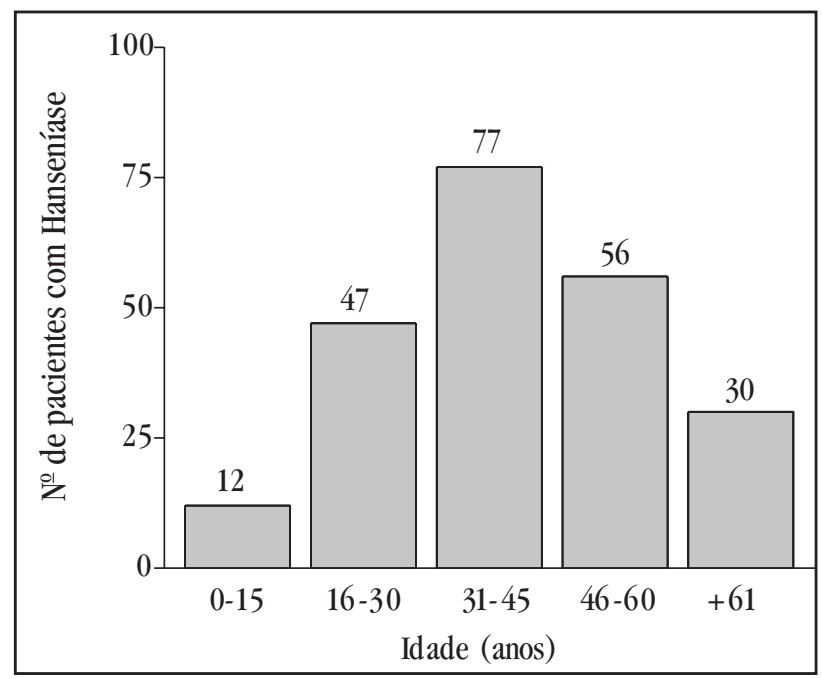

Figura 4 - Distribuição por faixa etária de casos novos de hanseníase, Prudentópolis-PR, 1998 a 2005.

\section{DISCUSSÃO}

Os achados do presente estudo confirmam que o número de casos novos de hanseníase em Prudentópolis vem se mantendo alta desde o período de 1998, considerado o município de maior da prevalência em casos de hanseníase no Paraná, nesse período. Além disto, no ano de 2002 foram detectados pelo menos duas vezes mais casos novos de hanseníase do que nos outros anos, tal fato ocorreu provavelmente devido, à intensificação de busca ativa de pacientes portadores da doença, por uma campanha do governo de busca ativa organizada pela Secretaria de Saúde do Estado do Paraná (SESA). Medida esta, que foi estabelecida pelo Comitê de Especialistas da Organização Mundial da Saúde (OMS) como estratégia global de eliminação da hanseníase ${ }^{8}$. Fato que demonstra que ainda há uma elevada necessidade da criação de novos projetos e linhas de combate a hanseníase, visto que medidas, como busca ativa, não resolveram o problema.

0 alto número de casos novos de hanseníase no ano de 2002, também foi descrita por outros autores como Moreira ${ }^{19} \mathrm{e}$ Opromolla e cols ${ }^{21}$, que relataram que somente no ano de 2002 houve um acréscimo no número de pacientes com hanseníase em várias regiões brasileiras avaliadas, tanto que a prevalência de hanseníase no Brasil nos anos de 2001 e 2002 foi de 4,4 casos por dez mil habitantes. Acredita-se que, esse fato tenha ocorrido devido à intensificação de busca ativa de comunicantes intradomiciliares nesse período. Atualmente, no Brasil, embora a hanseníase não tenha sido erradicada, o número de novos casos tem se mantido estável, mostrando que muitos casos novos irão surgir nos próximos anos. Um fato preocupante é essa prevalência oculta, definida como os casos novos esperados que não estejam sendo diagnosticadas ou o são tardiamente ${ }^{3}$.

Neste sentido, Opromolla e cols ${ }^{21}$ observaram que 0 comportamento de detecção de casos de hanseníase se mantém constante nos últimos 30 anos, constatando ainda que a distribuição da hanseníase em todo o Brasil ocorre de maneira desigual entre as áreas. Com isto, sugeriram que gestores conheçam a situação epidemiológica local para aí definirem ações prioritárias de acordo com cada situação ${ }^{11}$. Além disso, a hanseníase está fortemente relacionada às condições socioeconômicas, tais como: o crescimento acelerado da população dessas áreas, o deslocamento de contingente populacional de áreas rurais para as cidades, que pode não ter sido acompanhado de melhoria da estrutura urbana com conseqüente declínio nas condições de vida dessas populações ${ }^{12}$. E isto acontece na região de Prudentópolis, que é um município situado na região centro-sul do Estado do Paraná, possuindo uma área de $2.311 .601 \mathrm{~km}^{2}$, com uma população de 46.346 habitantes, sendo que 18.276 residem na área urbana e 28.070 na zona rural ${ }^{1314}$. Devido a grande extensão do município e a distância de algumas localidades da sede, a desinformação acaba gerando problemas sérios de saúde à população tais como a disseminação da hanseníase. Aliado a isso, o município também se encontra num quadro de extrema pobreza, cuja taxa de pobreza foi de 43,1\% e o Índice de Desenvolvimento Humano (IDH) de 0,733 , ocupando a posição 231 do total de 399 municípios no Estado do Paraná ${ }^{14}$.

Em 2005, no Paraná foi registrado uma taxa de prevalência de ponto-indicador para eliminação de hanseníase de aproximadamente 1,12/10.000 habitantes, considerada um índice de prevalência alto, enquanto que o Município de Prudentópolis apresentou um índice de 3,42/10.000 habitantes ${ }^{7}$. As razões para a continuada transmissão da hanseníase num contexto de ampla cobertura da PQT ainda não são bem compreendidas ${ }^{18}$. No entanto, alguns aspectos são importantes nesse contexto, como o longo período de incubação da doença, a evolução insidiosa e o preconceito da sociedade, são responsáveis pela 
omissão de informação no meio familiar e social, dificultando assim o conhecimento das fontes de infecção de casos novos de hanseníase, especialmente em áreas urbano-metropolitanas ${ }^{6}$. A forma da doença, bem como a proximidade do caso primário, também é um fator que contribui para o risco de adoecer. Ampliando os critérios da condição de contato para além daqueles domiciliares, a maioria dos casos incidentes poderia estar relacionada a um caso prévio de hanseníase, a exemplo da tuberculose. Os portadores de hanseníase e mesmo os profissionais de saúde freqüentemente não estão atentos a qualquer contato prévio com a doença, e na maioria dos casos novos não é possível relacionar com outros casos de hanseníase 6 . Fato que ocorreu no município de Prudentópolis, o qual não foi possível detectar fatores de risco para a infecção pelo bacilo Mycobacterium leprae como o contato com pacientes com a forma multibacilar, sem tratamento.

Neste aspecto, diversos estudos comprovam que os riscos são maiores nos contatos domiciliares de pacientes multibacilares (MB) do que com paucibacilares (PB) ${ }^{17}$. Esses dados podem explicar a alta incidência de hanseníase no município de Prudentópolis, visto que se observa nos dados, que a maioria (63\%) dos casos da doença, no município, apresenta a forma multibacilar (Figura 2). Além disto, 51\% dos casos totais de hanseníase se encontravam com a forma clínica transmissível da doença, virchowiana (Figura 3). Santos e cols ${ }^{23}$ avaliaram 175 pacientes com hanseníase no Estado de São Paulo, dos quais 134 (76,6\%) eram multibacilares, tendo o grupo virchowiano, com maior representatividade, sendo encontrado em 53,1\% (93 pacientes) dos casos. Goulart e cols ${ }^{10}$, também, avaliaram os prontuários de 187 pacientes de Uberlândia/MG e descreveram que $64,5 \%$ dos pacientes apresentavam a forma multibacilar, no entanto, com predomínio $(66,3 \%)$ da forma dimorfa.

Um dado importante mostrado nos resultados foi que a maioria dos pacientes com hanseníase no município faz parte da população economicamente ativa, entre a faixa etária de 31 a 45 anos (35\%), seguida de 46 a 60 anos (25\%), fato que pode constituir, portanto, num sério problema econômico para a região e gerar um imenso custo social. Um outro aspecto a considerar foram os dados que mostram predomínio das formas multibacilares e da faixa etária de 31 a 60 anos entre os pacientes, que podem ser explicados por um período de incubação mais prolongado da doença ou uma demora no diagnóstico ${ }^{21}$. Estudos similares realizados por Santos e cols ${ }^{18}$ também relataram predomínio de pacientes na faixa de 31-40 anos, seguindo a de 41-50 anos. Como em estudos anteriores, esses autores demonstraram que a incidência de hanseníase nas formas multibacilares cresceu com a idade, encontrando-se uma maior frequiência dessas formas da doença, quando comparada com as formas paucibacilares, em adultos com mais idade. Esses autores sugeriram que esse fato ocorra devido ao período de incubação das formas multibacilares serem mais longos que as formas paucibacilares ${ }^{24}$.

Por outro lado, outras investigações relataram que um dos indicadores mais sensíveis em relação à situação de controle da hanseníase é o percentual de casos em jovens relacionado à intensidade de transmissão da doença ${ }^{125}$. Neste sentido, Talhari e Neves propuseram que quanto maior a transmissão, maior a probabilidade do surgimento de hanseníase na população mais jovem, devido à maior facilidade de exposição ao bacilo de Hansen nos primeiros anos de vida. Por isto, é importante o monitoramento de áreas com ocorrência de casos novos em pessoas menores de 15 anos de idade, como indicador da magnitude do processo de transmissão da doença ${ }^{2}$. No entanto, neste estudo foram encontrados poucos casos de hanseníase em indivíduos entre a faixa etária de 0 a 15 anos (5,2\%), fato que pode ser explicado pela dificuldade em diagnosticar os sinais clínicos da hanseníase na infância ${ }^{23}$. Com isso, um controle rigoroso, envolvendo a vigilância epidemiológica deve ser mantido em crianças sob risco de contrair a hanseníase, no sentido de detectar a doença mais precocemente e evitar as consequiências do diagnóstico tardio e dos estigmas sociais ${ }^{4126}$.

A partir desses dados, pode-se concluir que o município de Prudentópolis apresentou um alto número de casosnovos de hanseníase, em indivíduos da faixa etária economicamente ativa e tendo a forma multibacilar ainda predominante na região. Esses indicadores apontam para uma elevada circulação do bacilo na comunidade, mostrando a dificuldade de se atingir à meta de eliminação da hanseníase, visto que existem obstáculos, como irregularidade e 0 abandono do tratamento pelos pacientes. Neste sentido, acreditamos queéde grande importância 0 aprimoramento das condições operacionais de rede de serviços e a necessidade da criação de novos projetos e linhas de combate à hanseníase no Brasil.

\section{REFERÊNCIAS}

1. Alves KR. Hanseníase em menores de 15 anos: perfil epidemiológico e clínicoevolutivo em residentes do Recife, de 1993 a 1997. Dissertação de Mestrado. Centro de Ciências da Saúde de Ciências da Saúde da Universidade Federal do Pernambuco, Recife, 1999.

2. Andrade V, Albuquerque MFM, Sabroza PC. The importance of operational factors for the interpretation of indicators in the Hansen's disease endemic in Brazil. Acta leprologica 10: 131-139, 1997.

3. Araújo MG. Hanseníase no Brasil. Revista da Sociedade Brasileira de Medicina Tropical 3: 373-382, 2003.

4. Bechelli LM, Dominguez VM, Patwary KM. WHO Epidemiologic random sample surveys of leprosy in Northern Nigeria (Katsina), Cameroon and Thailand (Khon Kaen). International Journal of Leprosy and Other Mycobacterial Diseases 34:223$243,1966$.

5. Duncan ME. Leprosy in young children: past, present and future. International Journal of Leprosy and Other Mycobacterial Diseases 3: 468-473, 1985.

6. Durães SMB, Guedes LS, Cunha MD, Cavaliere FAM, Oliveira MLWDR. Estudo de 20 focos familiares de hanseníase no município de Duque de Caxias, Rio de Janeiro, Anais Brasileiros de Dermatologia 80 (supl 3): S(295-300), 2005.

7. Ebenezer L, Arunthati S, Kurian N. Profile of leprosy in children: past and present. Indian Journal Leprosy 3: 255-259, 1997.

8. Fine PE, Sterne JA, Ponnighaus JM, Bliss L, Saui J, Chihana A. Household and dwelling contact as risk factors for leprosy in northern Malawi. American journal of Epidemiology 146:91-102, 1997.

9. Gillis TP, Krahenbuhl JL. Global elimination of leprosy. Reviews in Medical Microbiology 9: 39-48, 1998.

10. Goulart IMB. Efeitos adversos da poliquimioterapia em pacientes com hanseníase: um levantamento de cinco anos em um Centro de Saúde da Universidade Federal de Uberlândia. Revista da Sociedade Brasileira de Medicina Tropical 35: 453-460, 2002 . 
11. Hammond PJ, Sundar Rao PSSS. The tragedy of deformity in childhood leprosy. Leprosy Review 2: 217-220, 1999.

12. Helene LMF, Salum MJL. A reprodução social da hanseníase: um estudo do perfil de doentes com hanseníase no Município de São Paulo. Cadernos de Saúde Pública 1: 101-113, 2002.

13. Instituto Brasileiro de Geografia e Estatística. Disponível em http://www.ibge.gov. br/home/Acesso em 1 de julho de 2007.

14. Instituto Paranaense de Desenvolvimento Econômico e Social. Disponível em: http://www.ipardes.gov.br/index. Acesso em 1 de julho de 2007.

15. Lombardi C, Suàrez REG. Epidemiologia da hanseníase. In: Talhari S, Neves RG (eds) Hanseníase, $3^{\circ}$ edição, p.167, 1997.

16. Ministério da Saúde. Portaria $\mathrm{N}^{\circ} 1073 / \mathrm{GM}$ de 26 de setembro de 2000. Publicada no DOU - 188-E, p. 18 - Seção 1, 28 de setembro, 2000.

17. Ministério da Saúde. Hanseníase Atividades de controle e manual de procedimentos. Secretarias de Políticas Públicas, Brasília, 2001.

18. Ministério da Saúde. Vigilância Epidemiológica do Paraná, Cartas de Eliminação de Hanseníase $5^{\text {a }}$ edição. Disponível em: http:www.portal.saude.gov.br/portal, Brasília, 2006.
19. Moreira TA, Panorama sobre a hanseníase: quadro atual e perspectivas, História, Ciências, Saúde-Manguinhos 10: 291-307, 2003.

20. Noordeen SK. Elimination of leprosy as a public health problem: Progress and prospects. Bulletin of the World Health Organization 73: 1-6, 1995.

21. Opromolla PA, Dalben I, Cardim M. Análise da distribuição espacial da hanseníase no Estado de São Paulo, 1991-2002. Revista Brasileira de Epidemiologia 4: 356364, 2005.

22. Prasad PVS. Childhood Leprosy in a Rural Hospital. Indian Journal of Pediatrics 65: 751-754, 1998.

23. Santos GG, Marcucci G, Marchese LM, Guimarães Jr J. Aspectos estomatológicos das lesões específicas e não-específicas em pacientes portadores da moléstia de Hansen. Pesquisa Odontológica Brasileira 3: 268-272, 2000.

24. Souza WV, Barcellos CC, Brito AM, Carvalho MS, Cruz OG, Albuquerque MFM, Alvez KR, Lapa TM, Aplicação de modelo empírico na análise espacial da ocorrência de hanseníase. Revista de Saúde Pública 35: 474-480, 2001.

25. World Health Organization. Leprosy - Global situation. Site: http://www.who. int/wer. Weekly Epidemiological Record 735: 225-232. 2000.

26. World Health Organization. Global leprosy situation 2004. Available from: http:// www.who.int/wer/2005/en/wer8013.pdf, 2004. Weekly Epidemiological Record 13:118-124, 2005. Updated April 30, 2005. 